\title{
Prevalência de sobrepeso e obesidade entre funcionários plantonistas de unidades de saúde de Teresina, Piauí
}

\author{
Overweight and obesity prevalence in employees \\ of healthcare units in Teresina, Piauí, Brazil
}

Ruth Maria Rocha de Pádua SOUSA ${ }^{1}$

Débora Pereira SOBRAL ${ }^{2}$

Suzana M. Rebelo Sampaio da PAZ ${ }^{3}$

Maria do Carmo de Carvalho e MARTINS ${ }^{4}$

\section{RE S U M O}

\section{Objetivo}

O objetivo deste trabalho foi identificar a prevalência de sobrepeso e obesidade, bem como os fatores a eles associados, em funcionários de quatro Unidades de Saúde do Município de Teresina, Piauí.

\section{Métodos}

Estudo transversal com amostra estratificada proporcional de 207 funcionários plantonistas diurnos das Unidades Básicas de Saúde da Prefeitura Municipal de Teresina, que foram entrevistados e avaliados a partir de dados antropométricos. Para a classificação do estado nutricional foi utilizado o Índice de Massa Corporal e, para a classificação do risco cardiovascular de acordo com a distribuição da gordura corporal, foi utilizada a circunferência da cintura.

\section{Resultados}

A prevalência de excesso de peso foi de $53,72 \%$, sendo as proporções de sobrepeso e obesidade de $35,75 \%$ e $17,70 \%$, respectivamente. O sobrepeso esteve mais presente no sexo masculino $(45,16 \%)$ e a obesidade no sexo feminino $(19,31 \%)$. As variáveis que se mantiveram associadas significantemente com a obesidade foram: idade, função desempenhada pelo funcionário, obesidade dos pais, renda, fumo e circunferência da cintura.

\footnotetext{
1 Prefeitura Municipal de Teresina, Fundação Municipal de Saúde, Unidade de Saúde Buenos Aires. Teresina, PI, Brasil.

2 Prefeitura Municipal de Teresina, Fundação Municipal de Saúde, Unidade de Saúde Wall Ferraz. Teresina, PI, Brasil.

3 Universidade Federal do Piauí, Centro de Ciências da Saúde, Departamento de Medicina Comunitária. Teresina, PI, Brasil.

${ }^{4}$ Universidade Federal do Piauí, Centro de Ciências da Saúde, Departamento de Biofísica e Fisiologia. Campus Universitário Ministro Petrônio Portela, Bloco 8, 64049-660, Ininga, Teresina, PI, Brasil. Correspondência para/Correspondence to: M.C.C. MARTINS.E-mails:<mccm@ufpi.br>; <carminhacmartins@yahoo.com.br>.
} 
474 | R.M.R.P. SOUZA et al.

\section{Conclusão}

As elevadas proporções de excesso de peso entre os funcionários das Unidades de Saúde mostram a necessidade de ações de saúde destinadas à prevenção e ao controle, de modo a contribuir para a redução desses índices e para o controle das doenças crônicas.

Termos de indexação: índice de massa corporal; obesidade; sobrepeso.

\section{A B S T R A C T}

\section{Objective}

The objective of this study was to identify the prevalence of overweight and obesity and associated factors in employees of four Health Units in the municipality of Teresina, Piauí.

\section{Methods}

This was a cross-sectional study with proportionate stratified sampling of 207 day shift employees of Basic Healthcare Units of the city of Teresina, who were interviewed and classified according to their anthropometric data. Nutritional status was determined with body mass index and waist circumference was used to determine cardiovascular risk according to the distribution of body fat.

\section{Results}

The prevalence of excess weight was $53.72 \%$, with $35.16 \%$ overweight and $17.70 \%$ obese. Overweight was more common among males (45.16\%) and obesity among females (19.31\%). The variables that were significantly associated with obesity were: age, job, parental obesity, income, smoking and waist circumference.

\section{Conclusion}

The high rates of excess weight found among Healthcare Unit employees show that health actions to prevent and control obesity are needed to help reduce these indices and chronic disease rates.

Indexing terms: body mass index; obesity; overweight.

\section{N T R O D U Ç Ã O}

A obesidade, um dos distúrbios mais remotos que acomete a humanidade, é uma doença crônica que atinge todas as faixas etárias e grupos socioeconômicos ${ }^{1}$ e que, Segundo Coutinho², desponta como um problema mais freqüente e mais grave que a desnutrição, à medida que se consegue erradicar a miséria entre as camadas mais pobres da população. O aumento da prevalência de obesidade observado na região das Américas nas últimas décadas tem sido atribuído principalmente, às mudanças nos hábitos alimentares da população, com maior consumo de alimentos de alta densidade energética, alto consumo de carboidratos refinados, gorduras saturadas, colesterol, ácidos graxos trans, bebidas alcoólicas e alimentos tipo fast-food; redução da atividade física, devido aos avanços tecnológicos no trabalho, ao uso de veículos automotores, elevadores, escadas rolantes e aumento do tempo gasto em atividades sedentárias (jogos eletrônicos, televisão, computador); e a outros fatores de caráter sociocultural, como a valorização do excesso de peso como sinônimo de saúde e prosperidade 3 .

A preocupação com a obesidade reside no fato de que ela é, em si mesma, uma doença crônica, além de se constituir em reconhecido fator de risco para muitas outras doenças debilitantes e de alto custo social, como diabetes do tipo 2, hipertensão arterial, acidentes vasculares cerebrais, cardiopatias, dislipidemias e alguns tipos de câncer ${ }^{3,4}$. Desse modo, ao identificar sujeitos obesos também se identifica uma alta proporção dos sujeitos em risco de sofrer de outras doenças crônicas não transmissíveis ${ }^{3}$.

A distribuição da gordura corporal pode revelar alguma predisposição do indivíduo para o desenvolvimento de complicações. A gordura abdominal, por exemplo, pode elevar em até dez vezes o risco para o desenvolvimento de diabetes tipo 2 , sendo também fator de risco para hiper- 
tensão arterial em adultos com idade entre 20 e 45 anos $^{5}$.

Os países desenvolvidos têm concentrado esforços na área de Saúde Pública, com vistas à prevenção de doenças crônicas não transmissíveis. Para tanto, tem-se dado ênfase à redução da obesidade, à melhoria do padrão alimentar e ao combate ao sedentarismo. A implementação de ações que envolvem educação alimentar e nutricional e prática de exercícios físicos regulares pode ser de grande valia para a redução do contingente de obesos em todo o mundo.

No Brasil, a evolução do perfil antropométrico-nutricional da população, comparando estimativas da Pesquisa de Orçamentos Familiares $(\mathrm{POF})^{6}$ com dados da Pesquisa Nacional sobre Saúde e Nutrição (PNSN) ${ }^{7}$, mostrou que, no período de 1989 a 2002-2003, para a população geral, a prevalência de obesidade manteve-se estável (12,8\% na PNSN e $12,7 \%$ na POF). E, embora entre mulheres a evolução de excesso de peso e obesidade tenha mantido relativa estabilidade, na população masculina as prevalências de excesso de peso e obesidade aumentaram $(29,5 \%$ e $5,1 \%$ na PNSN e $41,0 \%$ e $8,8 \%$ na POF, respectivamente).

Vários trabalhos têm sido realizados no Brasil visando a estudar a freqüência de excesso de peso entre grupos populacionais, incluindo trabalhadores do serviço público. Frente à inexistência de registros conhecidos sobre o estudo de sobrepeso e obesidade entre funcionários públicos do Piauí, o propósito do presente estudo foi estimar a prevalência de sobrepeso e obesidade entre os funcionários públicos plantonistas das Unidades de Saúde da Fundação Municipal de Saúde de Teresina-Piauí, bem como identificar possíveis fatores associados ao excesso de peso e à distribuição de gordura corporal, com a perspectiva de fornecer dados que permitam a criação de programas de vigilância em saúde voltados para os servidores públicos municipais.

\section{MÉ T O D O S}

Foi realizado estudo transversal com amostra estratificada proporcional, constituída por funcionários plantonistas diurnos de quatro estratos representados por Unidades de Saúde (US) de Teresina, localizadas nas zonas Sul, Sudeste, Leste e Norte da cidade. Tais unidades foram selecionadas por figurarem entre as maiores do município, além de estarem localizadas em regiões distintas da cidade. A coleta dos dados foi realizada no período de julho a dezembro de 2004, no turno da manhã.

O cálculo do tamanho da amostra tomou como parâmetro a prevalência de excesso de peso de $32,00 \%$, revelada pela Pesquisa Nacional sobre Saúde e Nutrição (PNSN) ${ }^{7}$, realizada em 1989, gerando amostra igual a 210 funcionários. Para o estudo da prevalência de sobrepeso/obesidade, considerou-se aceitável um erro amostral de 5,00\% e nível de confiança de $95,00 \%$. Foram considerados critérios de exclusão: idade superior a 60 anos, gestação, e o fato de o funcionário estar substituindo o plantonista da Unidade de Saúde. A amostra foi escolhida por sorteio proporcional ao número de funcionários plantonistas na Unidade e no setor ou na função exercida. A amostra final foi composta por 207 participantes, sendo a perda igual a $0,48 \%$.

As informações foram obtidas por meio de um formulário de entrevista dividido em dados de identificação, dados socioeconômicos, antecedentes familiares e medidas antropométricas. $\mathrm{O}$ estudo foi previamente aprovado pela Comissão de Ética da Universidade Federal do Piauí e todos os entrevistados foram informados a respeito dos objetivos do trabalho e confirmaram a participação mediante assinatura de Termo de Consentimento Livre e Esclarecido, conforme prevê a Resolução 196/96 do Conselho Nacional de Saúde.

O sobrepeso e a obesidade foram definidos a partir do indice de massa corporal (IMC) e os critérios utilizados foram os propostos pela Organização Mundial de Saúde ${ }^{8}$, sendo consideradas obesas as pessoas que apresentaram IMC igual ou superior a $30 \mathrm{~kg} / \mathrm{m}^{2}$ e com sobrepeso aquelas com IMC entre 25 e $30 \mathrm{~kg} / \mathrm{m}^{2}$.

Foram estudadas as seguintes variáveis: idade (em anos completos), sexo, função desem- 
penhada na Unidade de Saúde, grau de instrução, situação conjugal (entrevistados que viviam com ou sem companheiro na época do estudo), renda familiar (renda mensal em salários mínimos de todos os moradores do domicílio, distribuída por faixas de 1 a 2, 2 a 5 e mais do que 5 salários mínimos), tabagismo (sendo considerados fumantes aqueles que fumavam qualquer quantidade de cigarro diariamente), exercício físico (distribuído em 4 grupos: não praticante de exercício físico, prática em até 3 vezes por semana, entre 3 e 6 vezes por semana, e diariamente), obesidade dos pais (referida pelo entrevistado), massa corporal (aferida em balança digital marca Plenna Litium com capacidade de $150 \mathrm{~kg}$ e variação de $0,1 \mathrm{~kg}$ ), estatura (coletada uma única vez com antropômetro de madeira construído exclusivamente para pesquisa de campo com precisão de $0,1 \mathrm{~cm}$ ) e circunferência da cintura (medida com fita métrica de fibra de vidro, em centímetros, com precisão de $1 \mathrm{~mm})$.

A circunferência da cintura (CC) foi utilizada com o objetivo de identificar o padrão de distribuição da massa adiposa, pois, segundo vários estudos, é o melhor indicador para a avaliação do risco de doenças cardiovasculares em estudos epidemiológicos ${ }^{9-12}$. A classificação da obesidade abdominal baseada na CC utilizou os pontos de corte recomendados pela Organização Mundial da Saúde ${ }^{8}$ para avaliação do risco de complicações metabólicas, que considera, para homens, risco aumentado valores de CC entre 94 e $102 \mathrm{~cm}$, e substancialmente aumentado valores de CC iguais ou superiores a $102 \mathrm{~cm}$; e para as mulheres, risco aumentado se CC entre 80 e $88 \mathrm{~cm}$, e muito aumentado para valores iguais ou superiores a $88 \mathrm{~cm}$.

As determinações de peso, altura e CC foram realizadas com os funcionários descalços, usando roupas leves e sem acessórios que pudessem interferir nas medidas, seguindo as recomendações do Manual de Técnicas e Procedimentos do Ministério da Saúde ${ }^{13}$. A CC foi verificada colocando-se a fita métrica inextensível ao nível da cintura natural (parte mais estreita do abdômen, logo abaixo da última costela), sem fazer pressão.

Os dados foram processados e analisados com o auxílio do programa Stata 7.014. As análises estatísticas foram realizadas utilizando como medidas de efeito proporções e respectivo erro-padrão. Foram também utilizados testes de associação do Qui-quadrado $\left(\chi^{2}\right)$ para tendência ao longo dos estratos e para estimar a associação entre potenciais fatores associados com sobrepeso e obesidade. Nos testes, a diferença foi considerada significante se $\alpha<5 \%$.

\section{RES U LTA D O S}

Foram pesquisados 207 funcionários adultos, sendo $57(27,54 \%)$ da Unidade de Saúde da zona sul, $55(26,57 \%)$ da zona leste, 52 $(25,12 \%)$ da zona norte e $43(20,77 \%)$ da zona sudeste. A maior parte dos funcionários era do sexo feminino (70,04\%). A idade variou entre 24 e 59 anos, sendo que $71,72 \%$ das mulheres tinham idade entre 40 e 59 anos e $75,80 \%$ dos homens encontravam-se entre 30 e 49 anos. Quanto à escolaridade, a maior parte dos funcionários havia cursado ensino médio e superior, sendo a função mais freqüente, para as mulheres, a de técnico/auxiliar de enfermagem e, para os homens, a de médico. Nessa perspectiva, os homens apresentaram renda maior, quando comparada com a das mulheres. Tanto os homens quanto as mulheres, em sua maioria, possuíam companheiros. Foi encontrada maior proporção de casados entre os homens.

Observou-se que as médias de peso, IMC e CC eram muito semelhantes entre os estratos e com a amostra geral.

$O$ excesso de peso $\left(\mathrm{IMC} \geq 25 \mathrm{~kg} / \mathrm{m}^{2}\right)$ foi encontrado em $53,72 \%\left(\mathrm{IC}_{95 \%} 46,58-60,56\right)$ dos funcionários pesquisados. O sobrepeso foi constatado em 35,75\% (IC $\left.{ }_{95 \%} 29,22-42,69\right)$ e a obesidade em $17,70 \%\left(\mathrm{IC}_{95 \%} 12,91-23,78\right)$ dos 
funcionários. Em relação ao sexo, as proporções de sobrepeso e obesidade encontradas foram, respectivamente, de $31,91 \%$ e $19,86 \%$ para o sexo feminino, e $43,94 \%$ e $13,64 \%$ para o sexo masculino. Observa-se, ainda, que as proporções de sobrepeso e obesidade são diferentes para homens na amostra geral e também no estrato 4 (Tabela 1).

A Tabela 2 mostra os fatores associados ao sobrepeso e à obesidade. A análise dos dados mostrou que a prevalência de obesidade aumentou com a idade $(p=0,037)$, observando-se que a proporção de obesos foi 3,6 vezes maior na faixa etária de 40 a 49 anos. Quanto ao sobrepeso, embora não tenha sido encontrada associação com a idade, sua freqüência foi 1,36 vezes maior nos funcionários de 30 anos e mais.

Com relação aos antecedentes familiares, a prevalência de obesidade foi cerca de 2 vezes maior nas pessoas que referiram obesidade da mãe e do pai do que entre aqueles cujos pais não eram, segundo sua opinião, obesos $(p=0,034)$.

Quando se estuda a estratificação por renda, verifica-se um decréscimo da prevalência de obesidade quando a renda mensal supera 5 salários mínimos $(p<0,0001)$, bem como relação inversa com a função exercida $(p<0,0001)$, ou seja, a obesidade é mais freqüente na função relacionada com mais baixo nível de escolaridade.

No presente estudo, o fumo mostra-se associado à obesidade $(p<0,01)$, encontrando-se maiores prevalências de obesidade entre ex-fumantes e não fumantes.
As prevalências de obesidade e sobrepeso aumentaram com a circunferência da cintura $(p<0,0001)$. A obesidade abdominal, que classifica o risco cardiovascular e de complicações metabólicas, revelou freqüências de risco aumentado em $28,02 \%$ e risco muito aumentado em $22,70 \%$ dos funcionários estudados. Observou-se, ainda, que o risco muito aumentado foi duas vezes mais freqüente para o sexo feminino. As Tabelas 3 e 4 mostram que não houve diferença significante no risco entre os indivíduos com sobrepeso dos diferentes estratos e em relação ao aumento da CC; no entanto, entre os obesos, o aumento da CC produziu aumento significante do risco nos estratos 2 e 4 e na amostra geral, quando se compara CC aumentada com muito aumentada.

\section{I S C U S S Ã O}

A análise dos dados antropométricos dos plantonistas das Unidades de Saúde municipais aqui estudados indicou alta freqüência de sobrepeso e obesidade, refletindo o quadro atual de prevalência de excesso de peso (IMC $\geq 25 \mathrm{~kg} / \mathrm{m}^{2}$ ) encontrado em outros estudos realizados no Brasil, especialmente, em alguns grupos de trabaIhadores. A prevalência de excesso de peso encontrada entre os plantonistas $(53,72 \%)$ foi maior que as encontradas por Abrantes et al. ${ }^{15}$ para a população adulta das regiões Nordeste e Sudeste, em 1997 (36,7\%) e na Pesquisa de Orçamento Familiar $^{6}$ de 2002-2003 para a população brasileira

Tabela 1. Prevalência de sobrepeso e obesidade segundo o sexo, por unidade de saúde (US) da rede pública municipal de Teresina, Piauí.

\begin{tabular}{|c|c|c|c|c|c|c|c|c|c|c|c|c|c|c|}
\hline & \multirow{3}{*}{$\mathrm{n}$} & \multirow{3}{*}{ C } & \multicolumn{5}{|c|}{ Homens } & \multirow{3}{*}{$n$} & \multirow{3}{*}{ C } & \multicolumn{5}{|c|}{ Mulheres } \\
\hline & & & \multicolumn{2}{|c|}{ Sobrepreso } & \multirow{2}{*}{ c } & \multicolumn{2}{|c|}{ Obesidade } & & & \multicolumn{2}{|c|}{ Sobrepreso } & \multirow{2}{*}{$C$} & \multicolumn{2}{|c|}{ Obesidade } \\
\hline & & & $\%$ & erro-padrão & & $\%$ & erro-padrão & & & $\%$ & erro-padrão & & $\%$ & erro-padrão \\
\hline US $1(n=43)$ & 13 & 6 & 46,15 & 13,83 & 4 & 30,77 & 12,80 & 30 & 10 & 33,33 & 8,61 & 4 & 13,33 & 6,21 \\
\hline US $2(n=52)$ & 11 & 5 & 45,45 & 15,01 & 2 & 18,18 & 11,63 & 41 & 14 & 34,15 & 7,41 & 8 & 19,51 & 6,19 \\
\hline US $3(n=57)$ & 20 & 7 & 35,00 & 10,66 & 2 & 10,00 & 6,71 & 37 & 11 & 29,73 & 7,51 & 6 & 16,22 & 6,06 \\
\hline US $4(n=55)$ & 18 & 10 & 55,60 & 11,71 & 1 & 5,56 & 5,40 & 37 & 11 & 29,73 & 7,51 & 10 & 27,03 & 7,30 \\
\hline Geral $(n=207)$ & 62 & 28 & 45,16 & 6,32 & 9 & 14,52 & 4,47 & 145 & 46 & 31,72 & 3,86 & 28 & 19,31 & 3,28 \\
\hline
\end{tabular}

n: número de funcionários no estrato; c: número de casos; teste de associação do Qui-quadrado por sexo segundo estrato; $p>0,05$. 
(cerca de 40,0\%), e ligeiramente inferior àquela encontrada em estudo realizado com funcionários de uma instituição de ensino superior $(59,9 \%)$ da cidade de Fortaleza-Ceará, em $1998^{16}$.
Os dados aqui apresentados mostraram que o excesso de peso foi mais freqüente no sexo masculino (59,68\%) do que no feminino (51,03\%), porém a obesidade foi mais prevalente no sexo

Tabela 2. Fatores associados ao sobrepeso e obesidade em funcionários plantonistas de Unidades de Saúde da rede pública municipal de Teresina, Piauí.

\begin{tabular}{|c|c|c|c|c|c|c|c|}
\hline \multirow{2}{*}{ Fatores de risco } & \multirow{2}{*}{$\mathrm{n}$} & \multicolumn{3}{|c|}{ Sobrepeso } & \multicolumn{3}{|c|}{ Obesidade } \\
\hline & & C & $\chi^{2}$ & $p$ & C & $\chi^{2}$ & $p$ \\
\hline \multicolumn{8}{|l|}{ Gênero } \\
\hline Masculino & 62 & 29 & \multirow{2}{*}{2,39} & \multirow{2}{*}{0,093} & 9 & \multirow{2}{*}{1,18} & \multirow{2}{*}{0,276} \\
\hline Feminino & 145 & 45 & & & 28 & & \\
\hline \multicolumn{8}{|l|}{ Idade (anos) } \\
\hline $20-29$ & 15 & 4 & \multirow{4}{*}{0,58} & \multirow{4}{*}{0,9} & 1 & \multirow{4}{*}{8,51} & \multirow{4}{*}{0,037} \\
\hline $30-39$ & 55 & 20 & & & 4 & & \\
\hline $40-49$ & 90 & 33 & & & 27 & & \\
\hline $50-59$ & 47 & 17 & & & 10 & & \\
\hline \multicolumn{8}{|l|}{ Função } \\
\hline Dentista & 7 & 4 & \multirow{6}{*}{4,74} & \multirow{6}{*}{0,447} & 1 & \multirow{6}{*}{17,16} & \multirow{6}{*}{0,004} \\
\hline Enfermeiro & 13 & 4 & & & 1 & & \\
\hline Médico & 52 & 23 & & & 1 & & \\
\hline Tecnico/Auxiliar Enfermagem & 66 & 22 & & & 16 & & \\
\hline Tecnico/Auxiliar Administração & 28 & 10 & & & 5 & & \\
\hline Auxiliar de Serviços & 41 & 11 & & & 13 & & \\
\hline \multicolumn{8}{|l|}{ Antecedentes familiares de obesidade } \\
\hline Nenhum & 143 & 54 & \multirow{3}{*}{1,43} & \multirow{3}{*}{0,489} & 19 & \multirow{3}{*}{6,75} & \multirow{3}{*}{0,034} \\
\hline Pai ou Mãe & 58 & 19 & & & 16 & & \\
\hline Pai e Mãe & 6 & 1 & & & 2 & & \\
\hline \multicolumn{8}{|l|}{ Situação Conjugal } \\
\hline Sem companheiro & 72 & 23 & 0695 & 0.404 & 11 & 0.507 & 0476 \\
\hline Com companheiro & 135 & 51 & 0,095 & 0,404 & 26 & 0,501 & $0,4 / 0$ \\
\hline Renda (salários mínimos) & & & & & & & \\
\hline 1 a 2 & 42 & 13 & & & 11 & & \\
\hline 2 a 5 & 75 & 26 & 3,636 & 0,162 & 23 & 17,735 & 0,000 \\
\hline$\geq 5$ & 80 & 35 & & & 3 & & \\
\hline Fumo & & & & & & & \\
\hline Fumante & 131 & 49 & & & 15 & & \\
\hline Não fumante & 31 & 8 & 1,57 & 0,456 & 8 & 10,38 & 0,006 \\
\hline Ex-fumante & 45 & 17 & & & 14 & & \\
\hline Exercício físico (n de dias/semana) & & & & & & & \\
\hline 0 & 137 & 46 & & & 24 & & \\
\hline Até 3 & 43 & 19 & 2,157 & 0,54 & 7 & 0,455 & 0,929 \\
\hline 3 a 6 & 32 & 8 & 2,101 & & 5 & & 0,525 \\
\hline 7 & 5 & 1 & & & 1 & & \\
\hline Circunferência da cintura (cm) & & & & & & & \\
\hline$<94$ ô ou $<80$ q & 104 & 19 & & & 2 & & \\
\hline entre 94 e 102 ơ ou entre 80 e 88 q & 56 & 38 & 38,97 & 0,000 & 5 & 88,697 & 0,000 \\
\hline$\geq 102$ ò ou $\geq 88$ q & 47 & 17 & & & 30 & & \\
\hline
\end{tabular}

n: número de funcionários; c: número de casos; $\chi^{2}$ : teste de associação do Qui-quadrado. 
Tabela 3. Risco cardiovascular pela circunferência da cintura para indivíduos com sobrepeso por unidade de saúde (US) municipal de Teresina-Piauí.

\begin{tabular}{|c|c|c|c|c|c|c|c|c|c|c|}
\hline \multirow{2}{*}{ Unidade de Saúde } & \multirow{2}{*}{$\mathrm{n}$} & \multicolumn{3}{|c|}{ Risco baixo } & \multicolumn{3}{|c|}{ Aumentado } & \multicolumn{3}{|c|}{ Muito aumentado } \\
\hline & & c & $\%$ & erro-padrão & c & $\%$ & erro-padrão & c & $\%$ & erro-padrão \\
\hline US 1 & 16 & 5 & 31,25 & 11,59 & 5 & 31,25 & 11,59 & 6 & 37,50 & 12,10 \\
\hline US 2 & 19 & 4 & 21,05 & 9,35 & 11 & 57,89 & 11,33 & 4 & 21,10 & 9,35 \\
\hline US 3 & 18 & 5 & 27,78 & 10,56 & 10 & 55,56 & 11,71 & 3 & 16,70 & 8,78 \\
\hline US 4 & 21 & 5 & 23,81 & 9,29 & 12 & 57,14 & 10,80 & 4 & 19,10 & 8,57 \\
\hline Geral & 74 & 19 & 25,68 & 5,08 & 38 & 51,53 & 5,81 & 17 & 23,00 & 4,89 \\
\hline
\end{tabular}

n: número de indivíduos com sobrepeso; c: número de casos; teste de associação do Qui-quadrado; p>0,05.

Tabela 4. Risco cardiovascular pela circunferência da cintura para indivíduos obesos segundo unidade de saúde (US) municipal de Teresina-Piauí.

\begin{tabular}{|c|c|c|c|c|c|c|c|c|c|c|}
\hline \multirow{2}{*}{ Unidade de Saúde } & \multirow{2}{*}{$n$} & \multicolumn{3}{|c|}{ Risco baixo } & \multicolumn{3}{|c|}{ Aumentado } & \multicolumn{3}{|c|}{ Muito aumentado } \\
\hline & & C & $\%$ & erro-padrão & c & $\%$ & erro-padrão & c & $\%$ & erro-padrão \\
\hline US 1 & 8 & 1 & 12,50 & 11,69 & 3 & 37,50 & 17,12 & 4 & 50,00 & 17,68 \\
\hline US 2 & 10 & - & - & - & 1 & 10,00 & 9,49 & 9 & 90,00 & 9,49 \\
\hline US 3 & 8 & - & - & - & 1 & 12,50 & 11,69 & 7 & 87,50 & 11,69 \\
\hline US 4 & 11 & 1 & 9,10 & 8,67 & - & - & - & 10 & 90,90 & 8,67 \\
\hline Geral & 37 & 2 & 5,41 & 3,72 & 5 & 13,51 & 5,62 & 30 & 81,08 & 6,44 \\
\hline
\end{tabular}

n: número de indivíduos com sobrepeso; c: número de casos; teste de associação do Qui-quadrado; p>0,05.

feminino (19,31\%). Concordantemente, em alguns estudos realizados com grupos de trabalhadores, as proporções de excesso de peso foram maiores nos homens que nas mulheres ${ }^{16,17}$. Ell et al. ${ }^{17}$, em estudo realizado com funcionários do Banco do Brasil no Estado do Rio de Janeiro, encontraram excesso de peso aproximadamente três vezes maior em homens do que em mulheres. Prevalência de excesso de peso significativamente maior no sexo masculino também foi encontrada no estudo de Sabry et al. ${ }^{16}$ entre funcionários de Universidade do Ceará.

Vários estudos realizados no Brasil e em outros países em desenvolvimento, têm mostrado que as mulheres têm maior probabilidade de apresentar obesidade do que os homens ${ }^{18,19}$. Entretanto, as maiores proporções de sobrepeso nos homens e de obesidade nas mulheres, encontradas no grupo de plantonistas, refletem a distribuição do excesso de peso na cidade de Teresina, onde foram encontradas, na POF de 2002-20036, prevalências de excesso de peso e obesidade de $41,5 \%$ e $7,8 \%$ para o sexo masculino e $36,3 \%$ e $9,5 \%$ para o sexo feminino.

Com relação aos fatores associados ao sobrepeso e à obesidade, segundo dados da POF 2002-2003 ${ }^{6}$, a relação da obesidade com a idade reproduz, em parte, o padrão para o excesso de peso, o qual tende a aumentar com a idade, de modo mais rápido para os homens (20-54 anos) e de modo mais lento e prolongado, para as mulheres (20-64 anos). Entre os funcionários das Unidades de Saúde de Teresina também houve aumento da prevalência de obesidade com o avançar da idade, porém não foi encontrada associação significante do sobrepeso com a idade. Contudo, no presente estudo, em virtude do tamanho da amostra e do critério de estratificação utilizado, não foi possível avaliar diferenças entre sexos para a variável idade, como também renda, função exercida na Unidade de Saúde, antecedentes familiares, tabagismo e prática regular de atividade física.

A prevalência de obesidade no grupo de plantonistas estudados, de acordo com o nível 
socioeconômico, mostra associação inversa, seguindo o padrão descrito para populações de países desenvolvidos, observando-se decréscimo da prevalência de obesidade quando a renda mensal supera 5 salários mínimos. Entretanto, outros estudos verificaram um incremento da prevalência de sobrepeso/obesidade com aumento de renda para homens, mas não para mulheres ${ }^{17,20}$. Observou-se, também, maior freqüência de casos na função relacionada com mais baixo nível de escolaridade.

Segundo Gigante et al..$^{20}$, a ocorrência de obesidade dos pais constituiu-se como um fator de risco para obesidade na população adulta de Pelotas, aumentando em duas vezes o risco de obesidade entre aqueles que referiram pai e mãe obesos. O estudo de fatores familiares nos funcionários das Unidades de Saúde de Teresina também mostrou associação com a obesidade, observando-se que a freqüência de obesidade foi 2,5 vezes maior entre aqueles cujos pais eram, segundo sua opinião, obesos.

Outros fatores associados à obesidade, encontrados no estudo de Gigante et al. ${ }^{20}$, incluem não fumar e não realizar exercício físico no lazer. A inatividade física, juntamente com o excesso de peso, a hipertensão arterial, a hipercolesterolemia, a dieta inadequada, o tabagismo e o consumo excessivo de álcool, constituem sete dos nove fatores de risco de maior relevância para as doenças crônicas não transmissíveis em países como o Brasil|21. Neste estudo, o hábito de fumar mostrou-se associado à obesidade $(p<0,01)$, encontrando-se maiores prevalências de obesidade entre ex-fumantes, seguida de não fumantes. Outros estudos também têm encontrado maiores prevalências de obesidade entre não fumantes e ex-fumantes.

Gigante et al. ${ }^{20}$ observaram prevalência de obesidade, respectivamente, de $24 \%$ e $23 \%$ para não fumantes e ex-fumantes, e de 14\% para fumantes na população adulta do município de Pelotas. E Raskin et al. ${ }^{22}$ encontraram associação entre tabagismo e índice de massa corpórea inferior a $30 \mathrm{~kg} / \mathrm{m}^{2}$ em mulheres climatéricas, observando número de pacientes não-obesas fumantes três vezes maior que o de obesas. Esses dados devem ser analisados com cautela, pois, embora exista uma espécie de senso comum de que o abandono do tabagismo provoca um aumento no ganho de peso, não há evidência conclusiva de ligação direta entre o tabagismo e a facilitação da perda de peso e a manutenção do peso corporal em estado estável, ou de grande efeito de parar de fumar sobre o ganho de peso corporal ${ }^{23}$. Além disso, mesmo que o efeito do fumo sobre o ganho de peso fosse, de fato, comprovado, ele seria menor que os benefícios associados aos hábitos de vida mais saudáveis, entre eles, o abandono do tabagismo.

Quanto à atividade física, apesar de não ter sido encontrada associação entre a prática regular de exercício físico com sobrepeso e obesidade no grupo de funcionários estudados, o elevado índice de sedentarismo encontrado (76,18\% dos funcionários não praticam nenhuma atividade física) constitui-se em um hábito insalubre, que necessita ser combatido e que tem sido relatado em outros grupos de trabalhadores. Nesse sentido, Nascimento \& Mendes ${ }^{24}$ observaram que mais da metade dos funcionários de um Centro de Saúde-Escola de Ribeirão Preto não praticava atividade física. Castro et al. ${ }^{25}$ constataram, em pesquisa, que mais da metade dos $44,6 \%$ de funcionários do sexo masculino de uma empresa metalúrgica do Rio de Janeiro, que apresentavam excesso de peso, não praticavam atividade física de lazer.

Embora existam motivos que justifiquem a utilização do IMC como indicador do estado nutricional em pesquisas epidemiológicas, seu uso deve ser cauteloso, visto que, apesar de o IMC $\geq 30 \mathrm{~kg} / \mathrm{m}^{2}$ ser utilizado como critério para definir obesidade, na verdade ele não mede o excesso de gordura corporal ${ }^{8,26}$. A despeito da possível superestimativa de prevalência de excesso de peso, resultados mostrando que mais da metade dos funcionários das Unidades de Saúde municipais apresentam algum grau de excesso de peso, justificam a necessidade de medidas de intervenção nutricional, com orientação individual. 
É amplamente conhecido que a distribuição central de adiposidade está associada a distúrbios metabólicos e a risco cardiovascular. As elevadas proporções de risco cardiovascular aumentado e muito aumentado encontradas no presente estudo, mediante utilização da circunferência da cintura, sugerem deposição visceral de gordura com perfil cardiovascular desfavorável no grupo estudado, em especial, nas mulheres.

A adiposidade central, indicando maior concentração de gordura intra-abdominal, está associada com sobrepeso e obesidade $(p<0,01)$ nos funcionários das Unidades de Saúde municipais. Embora a medida da circunferência da cintura seja referida em vários trabalhos como o melhor indicador para avaliação do risco cardiovascular $^{11}$, não existe ainda consenso acerca da definição dos pontos de corte para identificar adequadamente o risco de doenças cardiovasculares em grupos populacionais de diferentes etnias, especialmente considerando a diversidade da população brasileira' ${ }^{12}$. Neste estudo, a CC mostrou correlação positiva com IMC ( $r=0,87$ e $p=0,000)$, achados que estão de acordo com os de outras investigações ${ }^{10,11}$.

A proporção de funcionários das Unidades de Saúde municipais com sobrepeso ou com obesidade já instalada é preocupante, uma vez que representa elevado risco de morbidade, invalidez e mortalidade, com alto custo social. E, considerando-se que as condições de trabalho e de vida dos funcionários estudados assemelham-se àquelas de um segmento expressivo da população de Teresina e de todo o Estado do Piauí, onde boa parte da população é representada por funcionários públicos das redes municipal, estadual ou federal, os dados aqui apresentados interessam não só à Fundação Municipal de Teresina, como também à sociedade em geral, posto que apontam para a necessidade de vigilância nutricional desses trabalhadores. Nesse sentido, sugere-se a implantação de programas educacionais multidisciplinares com nutricionistas, educadores físicos, médicos e outros profissionais da saúde, a fim de orientar os trabalhadores de modo a incentivar hábitos de vida saudáveis, visando à promoção de qualidade de vida e à redução de ocorrência de doenças e de complicações evitáveis.

\section{REFERÊ NCIAS}

1. Organização Panamericana de Saúde. Doenças crônico-degenerativas e obesidade: uma estratégia mundial sobre alimentação saudável, atividade física e saúde. Brasília, 2003 [acesso em 13 dez 2004]. Disponível em: www.saúde.gov.br/bvs/ publicações

2. Coutinho WF. Consenso Latino-Americano em Obesidade. Arq Bras Endocrinol \& Metabol. 1999; 43(1):21-67.

3. Peña M, Bacallao J. La obesidad y sus tendencias en la región. Rev Panam Salud Pública. 2001; 10(2):75-8.

4. Field AE, Coakley EH, Must A, Spadano JL, Laird N, Dietz $\mathrm{WH}$, et al. Impact of overweight on the risk of developing common chronic disease during a 10-year period. Arch Int Med. 2001; 161(13): 1581-6.

5. Francischi RPP, Pereira LO, Freitas CS, Klopfer ML, Santos RC, Vieira P, et al. Obesidade: atualização sobre sua etiologia, morbidade e tratamento. Rev Nutr. 2000; 13(1):17-29.

6. Brasil. Ministério do Planejamento, Orçamento e Gestão. Instituto Brasileiro de Geografia e Estatística. Pesquisa de Orçamento Familiar 2002-2003. Análise da Disponibilidade Domiciliar de Alimentos e do Estado Nutricional no Brasil. Rio de Janeiro: IBGE; 2004. p.40-76.

7. Brasil. Instituto Nacional de Alimentação e Nutrição. Pesquisa Nacional Sobre Saúde e Nutrição de 1989. Condições nutricionais da população brasileira: adultos e Idosos. Brasília: INAN; 1991.

8. World Health Organization. Obesity: preventing and managing the global epidemic. Geneva; 1997. Report of a WHO consultation group on obesity.

9. Han TS, van Leer EM, Seidell JC, Lean ME. Waist circumference action levels in the identification of cardiovascular risk factors: prevalence study in a random sample. Br Med J. 1995; 311(7017):1401-5.

10. Lean ME, Han TS, Morrison CE. Waist circumference as a measure for indicating need for weight management. $\mathrm{Br}$ Med J. 1996; 311(15):158-61.

11. Molarius A, Seidell JC. Selection of anthropometric indicators for classification of abdominal fatness - a critical review. Int J Obes. 1998; 22(8):719-27. 
12. Velásquez-Meléndez G, Kac G, Valente JG, Tavares $R$, Silva CQ, Garcia SE. Evaluation of waist circumference to predict general obesity and arterial hypertension in women in Greater Metropolitan Belo Horizonte, Brazil. Cad Saúde Pública. 2002; 18(3):765-71.

13. Brasil. Ministério da Saúde. Universidade Federal de Goiás. Centro Colaborador em Alimentação e Nutrição da Região Centro-Oeste. Antropometria: manual de técnicas e procedimentos. Vigilância nutricional. 2a. ed. Goiânia; 2003.

14. Stata Statistical Software. Release 7.0 College Station. Texas: Stata Corporation; 2001.

15. Abrantes MA, Lamounier JA, Colosimo EA. Prevalência de sobrepeso e obesidade nas regiões nordeste e sudeste do Brasil. Rev Assoc Med Bras. 2003; 49(2):162-6.

16. Sabry MOD, Sampaio HAC, Silva MGC. Hipertensão e obesidade em um grupo populacional no Nordeste do Brasil. Rev Nutr. 2002; 15(2): 139-47.

17. Ell E, Camacho LAB, Chor D. Perfil antropométrico de funcionários de banco estatal no Estado do Rio de Janeiro/Brasil: I- Índice de massa corporal e fatores sócio-demográficos. Cad Saúde Pública. 1999; 15(1):113-21.

18. Sichieri R, Coitinho DC, Leão MM, Recine E, Everhart JE. High temporal, geographic, and income variation in body mass index among adults in Brazil. Am J Public Health. 1994; 84(5):793-8.

19. Monteiro CA, Mondini L, Souza ALM, Popkin BM. Da desnutrição para a obesidade: a transição nutricional no Brasil. In: Monteiro CA, organizador. Velhos e novos males da saúde no Brasil: a evo-lução do país e de suas doenças. São Paulo: Hucitec; 1995. p.247-55.

20. Gigante DP, Barros FC, Post CLA, Olinto MTA. Prevalência de obesidade em adultos e seus fatores de risco. Rev Saúde Pública. 1997; 31(3):236-46.

21. World Health Organization. Diet, nutrition and the prevention of chronic diseases. Geneva; 2003.

22. Raskin DBF, Pinto-Neto AM, Paiva LHSC, Raskin A, Martinez EZ. Fatores associados à obesidade e ao padrão andróide de distribuição da gordura corporal em mulheres climatéricas. Rev Bras Ginecol Obst. 2000; 22(7):435-41.

23. Gruber J, Frakes M. Does falling smoking lead to rising obesity? J Health Econ. 2006; 25(2): 183-97.

24. Nascimento LC, Mendes IJM. Perfil de saúde dos trabalhadores de um centro de saúde-escola. Rev Latino-Am Enfermagem. 2002; 10(4):502-8.

25. Castro MBT, Anjos LA, Lourenço PM. Padrão dietético e estado nutricional de operários de uma empresa metalúrgica do Rio de Janeiro, Brasil. Cad Saúde Pública. 2004; 20(4):926-34.

26. Anjos LA. Índice de massa corporal (massa corporal . estatura-2) como indicador do estado nutricional de adultos: revisão da literatura. Rev Saúde Pública. 1992; 26(6):431-6.

Recebido em: 29/11/2005

Versão final reapresentada em: 9/5/2007 Aprovado em: 13/7/2007 